\title{
Strong-weak convergence of two algorithms for total asymptotically nonexpansive mappings
}

\author{
Yan $\mathrm{Hao}^{\mathrm{a}, \mathrm{b}, *}$, Chaoping Wang ${ }^{\mathrm{a}, \mathrm{b}}$, Jie Zhou ${ }^{\mathrm{a}, \mathrm{b}}$ \\ a School of Mathematics, Physics and Information Science, Zhejiang Ocean University, Zhoushan 316022, China. \\ ${ }^{b}$ Key Laboratory of Oceanographic Big Data Mining and Application of Zhejiang Province, Zhoushan 316022, China. \\ Communicated by R. Saadati
}

\begin{abstract}
The purpose of this article is to investigate fixed point problems of total asymptotically nonexpansive mappings via two different iterative algorithms. We obtain strong and convergence theorems in the framework of Hilbert spaces. The main results improve and extend the recent corresponding results. (C)2017 All rights reserved.
\end{abstract}

Keywords: Hilbert space, convergence analysis, iterative algorithm, total asymptotically nonexpansive mappings, projection. 2010 MSC: 47H09, 47H10.

\section{Introduction and preliminaries}

Fixed point theory is dynamic and is experiencing an explosive growth in both theory and applications; as a consequence, research techniques and problems are drawn from various fields. As an important branch of nonlinear functional analysis and optimization theory, it has been applied to solve many real world problems, such as, the signal processing and the image reconstruction; see $[3,6,18]$ and the references therein. Fixed point algorithms are efficient methods for variational inequality problems, equilibrium problems, and saddle point problems; see $[4,8,9,17,19]$ and the references therein.

Let $\mathrm{H}$ be a real Hilbert space. Let $\mathrm{C}$ be a nonempty convex and closed subset of $\mathrm{H}$ and let $\mathrm{T}: \mathrm{C} \rightarrow \mathrm{C}$ be a mapping. Denote by $\operatorname{Fix}(\mathrm{T})$ the fixed point set of mapping $\mathrm{T}$. Recall that $\mathrm{T}$ is said to be nonexpansive iff

$$
\|T x-T y\| \leqslant\|x-y\|, \quad \forall x, y \in C .
$$

For the class of nonexpansive mappings, their fixed point sets may be empty, for example, let $\mathrm{H}=\mathrm{l}^{1}$ : all sequences $\left\{x_{n}\right\}$ such that $\sum\left|x_{n}\right|<\infty,\left\|x_{n}\right\|_{1}=\sum\left|x_{n}\right|$. Let $T: l^{1} \rightarrow l^{1}$ be the shift operator: $T x_{n}=$ $\left(0, x_{1}, x_{2}, \cdots\right), C:=\left\{\left\{x_{n}\right\}: x_{n} \geqslant 0,\left\|x_{n}\right\|_{1}=1\right\}$. Then $T: C \rightarrow C$ is a nonexpansive mapping without a fixed point. However, we know it always has an approximate fixed point if the convex and closed subset is also bounded in any Banach space.

\footnotetext{
*Corresponding author

Email address: zjhaoyan@yeah.net (Yan Hao)
}

doi:10.22436/jnsa.010.05.36 
$T$ is said to be asymptotically nonexpansive iff there exists a positive sequence $\left\{h_{n}\right\} \subset[1, \infty)$ with $\lim _{n \rightarrow \infty} h_{n}=1$ such that

$$
\left\|T^{n} x-T^{n} y\right\| \leqslant h_{n}\|x-y\|, \quad \forall x, y \in C, n \geqslant 1 .
$$

The class of asymptotically nonexpansive mappings was introduced by Goebel and Kirk [11] as a generalization of the class of nonexpansive mappings. If $\mathrm{C}$ is a nonempty closed convex and bounded subset of $\mathrm{H}$, then the set of fixed points of $\mathrm{T}$ is not empty. Indeed, the framework of the space can also be extended to the framework of uniformly convex Banach spaces; see [11] and the references therein.

$\mathrm{T}$ is said to be asymptotically nonexpansive in the intermediate sense iff it is continuous and the following inequality holds:

$$
\limsup _{n \rightarrow \infty} \sup _{x, y \in C}\left(\left\|T^{n} x-T^{n} y\right\|-\|x-y\|\right) \leqslant 0
$$

Observe that if we define

$$
\sigma_{n}=\sup _{x, y \in C}\left(\left\|T^{n} x-T^{n} y\right\|-\|x-y\|\right) \quad \text { and } v_{n}=\max \left\{0, \sigma_{n}\right\},
$$

then $v_{n} \rightarrow 0$ as $n \rightarrow \infty$. It follows that (1.1) is reduced to

$$
\left\|T^{n} x-T^{n} y\right\| \leqslant\|x-y\|+v_{n}, \quad \forall x, y \in C, n \geqslant 1 .
$$

The class of mappings which are asymptotically nonexpansive in the intermediate sense was introduced by Bruck et al. [5]. It is worth mentioning that the class of mappings which are asymptotically nonexpansive in the intermediate sense, contains properly the class of asymptotically nonexpansive mappings. It is known that the class of mappings which are asymptotically nonexpansive in the intermediate sense may not be Lipschitz continuous.

$T$ is said to be generalized asymptotically nonexpansive iff there exists positive sequences $\left\{h_{n}\right\} \subset[1, \infty)$ and $\left\{v_{n}\right\} \subset[0, \infty)$ with $\lim _{n \rightarrow \infty} v_{n}=0$ such that

$$
\left\|T^{n} x-T^{n} y\right\| \leqslant h_{n}\|x-y\|+v_{n}, \quad \forall x, y \in C, n \geqslant 1 .
$$

The class of generalized asymptotically nonexpansive mappings was introduced by Agarwal et al. [1] as a generalization of the class of nonexpansive mappings. They obtained weak convergence of an implicit two-step iterative algorithm in a Banach space; see [1] and the references therein.

Recently, Alber et al. [2] introduced the conception of total asymptotically nonexpansive mappings. Recall that $T$ is said to be total asymptotically nonexpansive iff

$$
\left\|T^{n} x-T^{n} y\right\| \leqslant\|x-y\|+\mu_{n} \psi(\|x-y\|)+v_{n}, \quad \forall x, y \in C,
$$

where $\psi:[0, \infty) \rightarrow[0, \infty)$ is strictly increasing continuous function with $\psi(0)=0$ and $\left\{\mu_{n}\right\}$ and $\left\{v_{n}\right\}$ are nonnegative real sequences such that $\mu_{n} \rightarrow 0$ and $\nu_{n} \rightarrow 0$ as $n \rightarrow \infty$. From the definition, we see that the class of total asymptotically nonexpansive mappings includes the class of asymptotically nonexpansive mappings as a special case; see [2] for more details.

Recently, the study of the convergence analysis of various iterative algorithms for solving various nonlinear mathematical models forms the major part of numerical mathematics. Among these iterative processes, Mann iterative algorithm and Ishikawa iterative algorithm are popular and hot. Let $C$ be a nonempty, closed, and convex subset of a real Hilbert space $\mathrm{H}$, and let $\mathrm{T}: \mathrm{C} \rightarrow \mathrm{C}$ be a nonexpansive mapping. Mann iterative algorithm generates a sequence $\left\{x_{n}\right\}$ in the following manner:

$$
x_{0} \in C, \quad x_{n+1}=\alpha_{n} T x_{n}+\left(1-\alpha_{n}\right) x_{n}, \quad \forall n \geqslant 0,
$$

where $\left\{\alpha_{n}\right\}$ is a real sequence in $(0,1)$. In 1979, Reich [21] obtained a celebrated weak convergence theorem in the framework of Banach spaces; see [21] and the references therein for more details. 
Recall that the modified Mann iteration which was introduced by Schu [23] generates a sequence $\left\{x_{n}\right\}$ in the following manner:

$$
x_{1} \in C, \quad x_{n+1}=\left(1-\alpha_{n}\right) x_{n}+\alpha_{n} T^{n} x_{n}, \quad \forall n \geqslant 1,
$$

where $\left\{\alpha_{\mathfrak{n}}\right\}_{\mathfrak{n}=0}^{\infty}$ is a sequence in $(0,1)$ and $\mathrm{T}: \mathrm{C} \rightarrow \mathrm{C}$ is an asymptotically nonexpansive mapping. In 1991, Schu [23] obtained the two strong convergence theorems with the aid of compactness, see [23] for more details.

Recall that the Ishikawa iterative algorithm generates a sequence $\left\{x_{n}\right\}$ in the following manner:

$$
\left\{\begin{array}{l}
x_{0} \in C, \\
y_{n}=\beta_{n} T x_{n}+\left(1-\beta_{n}\right) x_{n}, \\
x_{n+1}=\alpha_{n} T y_{n}+\left(1-\alpha_{n}\right) x_{n}, \quad \forall n \geqslant 0,
\end{array}\right.
$$

where $\left\{\alpha_{n}\right\}$ and $\left\{\beta_{n}\right\}$ are real sequences in $(0,1)$ and $T$ is a nonexpansive mapping. Since 1974, the twostep iterative algorithm has been extensively studied by many authors; see [12,14] and the references therein.

Recall that the modified Ishikawa iterative process which was introduced by Schu [22], $\left\{x_{n}\right\}$ in the following manner:

$$
\left\{\begin{array}{l}
x_{0} \in C \\
y_{n}=\beta_{n} T^{n} x_{n}+\left(1-\beta_{n}\right) x_{n}, \\
x_{n+1}=\alpha_{n} T^{n} y_{n}+\left(1-\alpha_{n}\right) x_{n}, \quad \forall n \geqslant 0,
\end{array}\right.
$$

where $\left\{\alpha_{n}\right\}$ and $\left\{\beta_{n}\right\}$ are real sequences in $(0,1)$ and $T$ is an asymptotically nonexpansive mapping in $C$.

The (modified) Ishikawa iterative algorithm is indeed more general than the (modified) Mann iterative algorithm. But research has been concentrated on the latter due probably to the reasons that the formulation of the (modified) Mann iterative algorithm is simpler than that of the (modified) Ishikawa iterative algorithm and that a convergence theorem for the (modified) Mann iterative algorithm may possibly lead to a convergence theorem for the (modified) Ishikawa iterative algorithm provided the control sequences satisfy certain appropriate conditions; see [13] and the references therein. We also remark here that both the (modified) Ishikawa iterative algorithm and the (modified) Mann iterative algorithm are only weakly convergent; see [10, 22]. In many fields, problems arise in infinite dimension spaces. In such problems, strong convergence is often much more desirable than weak convergence. Recently, many authors have extensively studied the strong convergence analysis of the different modified Ishikawa and Mann iterative algorithms; see [7, 13, 14, 20, 25] and the references therein.

Based on the modified Ishikawa iterative algorithm, we investigate the following two-step iterative algorithm for a pair of total asymptotically nonexpansive mappings $S$ and $T$,

$$
\left\{\begin{array}{l}
x_{0} \in C, \\
y_{n}=\beta_{n} S^{n} x_{n}+\left(1-\beta_{n}\right) x_{n}, \\
x_{n+1}=\alpha_{n} T^{n} y_{n}+\left(1-\alpha_{n}\right) x_{n}, \quad \forall n \geqslant 0,
\end{array}\right.
$$

where $\left\{\alpha_{n}\right\}$ and $\left\{\beta_{n}\right\}$ are real sequences in $(0,1)$. We establish strong and weak convergence theorems of common fixed points of a pair of total asymptotically nonexpansive mappings in the framework of Hilbert spaces.

To obtain our main convergence results, we need the following tools.

Recall that a space satisfies the Opial's condition [16] iff for any sequence $\left\{x_{n}\right\}$ in $H$ with the fact that $\left\{x_{n}\right\}$ converges weakly to $x, \liminf _{n \rightarrow \infty}\left\|x_{n}-x\right\|<\liminf _{n \rightarrow \infty}\left\|x_{n}-y\right\|$ for any $y \neq x$. Indeed, it is also equivalent to $\limsup _{n \rightarrow \infty}\left\|x_{n}-x\right\|<\limsup _{n \rightarrow \infty}\left\|x_{n}-y\right\|$ for any $y \neq x$.

Lemma 1.1 ([24]). Let $\left\{r_{n}\right\},\left\{s_{n}\right\}$, and $\left\{t_{n}\right\}$ be three nonnegative sequences satisfying the following condition:

$$
\mathrm{r}_{\mathrm{n}+1} \leqslant\left(1+\mathrm{s}_{\mathrm{n}}\right) \mathrm{r}_{\mathrm{n}}+\mathrm{t}_{\mathrm{n}}, \quad \forall \mathrm{n} \geqslant \mathrm{n}_{0},
$$

where $n_{0}$ is some nonnegative integer. If $\sum_{n=1}^{\infty} s_{n}<\infty$ and $\sum_{n=1}^{\infty} t_{n}<\infty$, then $\lim _{n \rightarrow \infty} r_{n}$ exists. 
Lemma 1.2 ([23]). Let $0<p \leqslant t_{n} \leqslant q<1$ for all $n \geqslant 1$. Suppose that $\left\{x_{n}\right\}$ and $\left\{y_{n}\right\}$ are sequences in $H$ such that

$$
\lim _{n \rightarrow \infty}\left\|t_{n} x_{n}+\left(1-t_{n}\right) y_{n}\right\|=d,
$$

$\limsup _{n \rightarrow \infty}\left\|x_{n}\right\| \leqslant d$, and $\limsup _{n \rightarrow \infty}\left\|y_{n}\right\| \leqslant d$ hold for some $r \geqslant 0$. Then $\left\|y_{n}-x_{n}\right\| \rightarrow 0$ as $n \rightarrow \infty$.

\section{Weak convergence theorems}

Theorem 2.1. Let $\mathrm{C}$ be a nonempty bounded closed and convex subset of a real Hilbert space $\mathrm{H}$. Let $\mathrm{T}: \mathrm{C} \rightarrow \mathrm{C}$ be a uniformly $\mathrm{L}_{\mathrm{t}}$-Lipschitz and total asymptotically nonexpansive mapping with function $\psi_{\mathrm{t}}$ and nonnegative real sequences $\left\{\mu_{n, t}\right\}$ and $\left\{v_{n, t}\right\}$ such that $\sum_{n=0}^{\infty} \mu_{n, t}<\infty$ and $\sum_{n=0}^{\infty} v_{n, t}<\infty$. Let $S: C \rightarrow C$ be a uniformly $L_{s^{-}}$ Lipschitz and total asymptotically nonexpansive mapping with function $\psi_{\mathrm{s}}$ and nonnegative real sequences $\left\{\mu_{\mathrm{n}, \mathrm{s}}\right\}$ and $\left\{v_{n, s}\right\}$ such that $\sum_{n=0}^{\infty} \mu_{n, s}<\infty$ and $\sum_{n=0}^{\infty} v_{n, s}<\infty$. Assume $\operatorname{Fix}(T) \cap \operatorname{Fix}(S) \neq \emptyset$ and let $\left\{x_{n}\right\}$ be a sequence generated by the following manner:

$$
\left\{\begin{array}{l}
x_{0} \in C \\
y_{n}=\left(1-\beta_{n}\right) x_{n}+\beta_{n} S^{n} x_{n}, \\
x_{n+1}=\left(1-\alpha_{n}\right) x_{n}+\alpha_{n} T^{n} y_{n}, \quad \forall n \geqslant 0
\end{array}\right.
$$

where $\left\{\alpha_{n}\right\}$ and $\left\{\beta_{n}\right\}$ are two sequences in $(0,1)$ such that $0<a \leqslant \alpha_{n} \leqslant a^{\prime}<1$ and $0<b \leqslant \beta_{n} \leqslant b^{\prime}<1$, where $a, a^{\prime}, b$, and $b^{\prime}$ are four real constants. Then $\left\{x_{n}\right\}$ converges weakly to a common fixed point of $T$ and $S$.

Proof. Fixing $p \in \operatorname{Fix}(T) \cap \operatorname{Fix}(S)$, we see that

$$
\begin{aligned}
\left\|y_{n}-p\right\| & \leqslant\left(1-\beta_{n}\right)\left\|x_{n}-p\right\|+\beta_{n}\left\|S^{n} x_{n}-p\right\| \\
& \leqslant\left(1-\beta_{n}\right)\left\|x_{n}-p\right\|+\beta_{n}\left(\left\|x_{n}-p\right\|+\mu_{n, s} \psi_{s}\left(\left\|x_{n}-p\right\|\right)+v_{n, s}\right) \\
& \leqslant\left\|x_{n}-p\right\|+\beta_{n} \mu_{n, s} \psi_{s}\left(\left\|x_{n}-p\right\|\right)+\beta_{n} v_{n, s} .
\end{aligned}
$$

This implies that

$$
\begin{aligned}
\left\|x_{n+1}-p\right\| \leqslant & \left(1-\alpha_{n}\right)\left\|x_{n}-p\right\|+\alpha_{n}\left(\left\|y_{n}-p\right\|+\mu_{n, t} \psi_{t}\left(\left\|y_{n}-p\right\|\right)+v_{n, t}\right) \\
\leqslant & \left(1-\alpha_{n}\right)\left\|x_{n}-p\right\|+\alpha_{n}\left(\left\|x_{n}-p\right\|+\beta_{n} \mu_{n, s} \psi_{s}\left(\left\|x_{n}-p\right\|\right)+\beta_{n} v_{n, s}\right) \\
& +\alpha_{n} \mu_{n, t} \psi_{t}\left(\left\|y_{n}-p\right\|\right)+\alpha_{n} v_{n, t} \\
\leqslant & \left\|x_{n}-p\right\|+\alpha_{n} \beta_{n} \mu_{n, s} \psi_{s}\left(\left\|x_{n}-p\right\|\right)+\alpha_{n} \beta_{n} v_{n, s}+\alpha_{n} \mu_{n, t} \psi_{t}\left(\left\|y_{n}-p\right\|\right)+\alpha_{n} v_{n, t} \\
\leqslant & \left\|x_{n}-p\right\|+\mu_{n, s} \psi_{s}(\operatorname{diam} C)+\mu_{n, t} \psi_{t}(\operatorname{diam} C)+v_{n, t}+v_{n, s} .
\end{aligned}
$$

Since $\sum_{n=0}^{\infty} \mu_{n, s}<\infty, \sum_{n=0}^{\infty} v_{n, s}<\infty, \sum_{n=0}^{\infty} \mu_{n, t}<\infty$, and $\sum_{n=0}^{\infty} v_{n, t}<\infty$, we obtain from Lemma 1.1 that $\lim _{n \rightarrow \infty}\left\|x_{n}-p\right\|$ exists. Next, we assume that $\lim _{n \rightarrow \infty}\left\|x_{n}-p\right\|=d>0$. Note that

$$
\left\|y_{n}-p\right\| \leqslant\left\|x_{n}-p\right\|+\mu_{n, s} \psi_{s}\left(\left\|x_{n}-p\right\|\right)+v_{n, s} .
$$

Hence, we have $\limsup _{n \rightarrow \infty}\left\|y_{n}-p\right\| \leqslant d$. On the other hand, we have

$$
\left\|T^{n} y_{n}-p\right\| \leqslant\left\|y_{n}-p\right\|+\mu_{n, t} \psi_{t}\left(\left\|y_{n}-p\right\|\right)+v_{n, t} \leqslant\left\|y_{n}-p\right\|+\mu_{n, t} \psi_{t}(\operatorname{diam} C)+v_{n, t} .
$$

This implies that $\limsup _{n \rightarrow \infty}\left\|T^{n} y_{n}-p\right\| \leqslant d$. Since

$$
\left\|x_{n+1}-p\right\|=\left\|\left(1-\alpha_{n}\right)\left(x_{n}-p\right)+\alpha_{n}\left(T^{n} y_{n}-p\right)\right\|,
$$

we find from Lemma 1.2 that

$$
\lim _{n \rightarrow \infty}\left\|T^{n} y_{n}-x_{n}\right\|=0
$$


From (2.1), we have

$$
\left\|x_{n}-p\right\| \leqslant\left\|x_{n}-T^{n} y_{n}\right\|+\left\|T^{n} y_{n}-p\right\| \leqslant\left\|x_{n}-T^{n} y_{n}\right\|+\left\|y_{n}-p\right\|+\mu_{n, t} \psi_{t}(\operatorname{diam} C)+v_{n, t} .
$$

In view of (2.2), we find that $\liminf _{n \rightarrow \infty}\left\|y_{n}-p\right\| \geqslant d$, which in turn implies that $\lim _{n \rightarrow \infty}\left\|y_{n}-p\right\|=d$. Since

$$
\left\|S^{n} x_{n}-p\right\| \leqslant\left\|x_{n}-p\right\|+\mu_{n, s} \psi_{s}\left(\left\|x_{n}-p\right\|\right)+v_{n, s} \leqslant\left\|x_{n}-p\right\|+\mu_{n, s} \psi_{s}(\operatorname{diam} C)+v_{n, s},
$$

we have $\limsup _{n \rightarrow \infty}\left\|S^{n} x_{n}-p\right\| \leqslant d$. On the other hand, we have

$$
\left\|y_{n}-p\right\|=\left\|\left(1-\beta_{n}\right)\left(x_{n}-p\right)+\beta_{n}\left(S^{n} x_{n}-p\right)\right\| .
$$

Using Lemma 1.2, we find that

$$
\lim _{n \rightarrow \infty}\left\|S^{n} x_{n}-x_{n}\right\|=0
$$

Note that

$$
\begin{aligned}
\left\|T^{n} x_{n}-x_{n}\right\| & \leqslant\left\|T^{n} x_{n}-T^{n} y_{n}\right\|+\left\|T^{n} y_{n}-x_{n}\right\| \\
& \leqslant L_{t}\left\|x_{n}-y_{n}\right\|+\left\|T^{n} y_{n}-x_{n}\right\| \\
& \leqslant L_{t}\left\|S^{n} x_{n}-x_{n}\right\|+\left\|T^{n} y_{n}-x_{n}\right\| .
\end{aligned}
$$

From (2.2) and (2.3), we have

$$
\lim _{n \rightarrow \infty}\left\|T^{n} x_{n}-x_{n}\right\|=0
$$

It also follows from (2.2) that

$$
\lim _{n \rightarrow \infty}\left\|x_{n+1}-x_{n}\right\|=0 .
$$

In view of the following two inequalities

$$
\left\|T x_{n}-x_{n}\right\| \leqslant\left\|x_{n}-x_{n+1}\right\|+\left\|x_{n+1}-T^{n+1} x_{n+1}\right\|+\left\|T^{n+1} x_{n+1}-T^{n+1} x_{n}\right\|+\left\|T^{n+1} x_{n}-T x_{n}\right\|,
$$

and

$$
\left\|S x_{n}-x_{n}\right\| \leqslant\left\|x_{n}-x_{n+1}\right\|+\left\|x_{n+1}-S^{n+1} x_{n+1}\right\|+\left\|S^{n+1} x_{n+1}-S^{n+1} x_{n}\right\|+\left\|S^{n+1} x_{n}-S x_{n}\right\|,
$$

we find from (2.3), (2.4), and (2.5) that

$$
\lim _{n \rightarrow \infty}\left\|T x_{n}-x_{n}\right\|=0,
$$

and

$$
\lim _{n \rightarrow \infty}\left\|S x_{n}-x_{n}\right\|=0 .
$$

Since $C$ is bounded, there exists a subsequence $\left\{x_{n_{i}}\right\}$ of $\left\{x_{n}\right\}$ such that $x_{n_{i}} \rightarrow \bar{x}$. Fix $k \in\left(0, \frac{1}{1+L_{s}}\right)$ and define $y_{\kappa, \rho}=k S^{\rho} \bar{x}+(1-\kappa) \bar{x}$ for arbitrary but fixed $\rho \geqslant 1$. Since both the mappings $S$ and T are Lipschitz continuous, we have

$$
\left\|S^{\rho} x_{n}-x_{n}\right\| \leqslant\left\|S x_{n}-x_{n}\right\|+\left\|S^{2} x_{n}-S x_{n}\right\|+\cdots+\left\|S^{\rho} x_{n}-S^{\rho-1} x_{n}\right\| \leqslant L_{s} k\left\|S x_{n}-x_{n}\right\| .
$$

It follows from (2.7) that

$$
\lim _{n \rightarrow \infty}\left\|S^{\rho} x_{n}-x_{n}\right\|=0
$$

Note that

$$
\begin{aligned}
\left\langle S^{\rho} y_{\kappa, \rho}-y_{\kappa, \rho}, y_{\kappa, \rho}-\bar{x}\right\rangle & =\left\langle x_{n}-y_{\kappa, \rho}, y_{\kappa, \rho}-S^{\rho} y_{\kappa, \rho}\right\rangle+\left\langle\bar{x}-x_{n}, y_{\kappa, \rho}-S^{\rho} y_{\kappa, \rho}\right\rangle \\
& =\left\langle x_{n}-y_{\kappa, \rho}, S^{\rho} x_{n}-S^{\rho} y_{\kappa, \rho}\right\rangle+\left\langle\bar{x}-x_{n}, y_{\kappa, \rho}-S^{\rho} y_{\kappa, \rho}\right\rangle
\end{aligned}
$$




$$
\begin{aligned}
& -\left\langle x_{n}-y_{\kappa, \rho}, x_{n}-y_{\kappa, \rho}\right\rangle+\left\langle x_{n}-y_{\kappa, \rho}, x_{n}-S^{\rho} x_{n}\right\rangle \\
\leqslant & \left\langle x_{n}-\bar{x}, S^{\rho} y_{\kappa, \rho}-y_{\kappa, \rho}\right\rangle \\
& +\left\|x_{n}-y_{\kappa, \rho}\right\|\left(\left\|x_{n}-y_{\kappa, \rho}\right\|+\mu_{\rho, s} \psi_{s}\left(\left\|x_{n}-y_{\kappa, \rho}\right\|\right)+v_{\rho, s}\right) \\
& -\left\|x_{n}-y_{k, \rho}\right\|^{2}+\left\|x_{n}-y_{\kappa, \rho}\right\|\left\|x_{n}-S^{\rho} x_{n}\right\| \\
\leqslant & \left\langle\bar{x}-x_{n}, y_{\kappa, \rho}-S^{\rho} y_{\kappa, \rho}\right\rangle+\left\|x_{n}-y_{\kappa, \rho}\right\|\left\|x_{n}-S^{\rho} x_{n}\right\| \\
& +\mu_{\rho, s}(\operatorname{diam} C) \psi_{s}((\operatorname{diam} C))+(\operatorname{diam} C) v_{\rho, s} .
\end{aligned}
$$

Using (2.8), we have

$$
\left\langle\bar{x}-y_{\kappa, \rho}, y_{\kappa, \rho}-S^{\rho} y_{\kappa, \rho}\right\rangle \leqslant \mu_{\rho, s}(\operatorname{diam} C) \psi_{s}((\operatorname{diam} C))+(\operatorname{diam} C) v_{\rho, s} .
$$

On the other hand, we have

$$
\begin{aligned}
\kappa\left\|\bar{x}-S^{\rho} \bar{x}\right\|^{2} & =\left\langle\bar{x}-y_{\kappa, \rho}, \bar{x}-S^{\rho} \bar{x}\right\rangle \\
& =\left\langle\bar{x}-y_{\kappa, \rho},\left(\bar{x}-S^{\rho} \bar{x}\right)-\left(y_{\kappa, \rho}-S^{\rho} y_{\kappa, \rho}\right)\right\rangle+\left\langle\bar{x}-y_{\kappa, \rho}, y_{\kappa, \rho}-S^{\rho} y_{\kappa, \rho}\right\rangle \\
& \leqslant\left(L_{s}+1\right) \kappa^{2}\left\|S^{\rho} \bar{x}-\bar{x}\right\|^{2}+\left\langle\bar{x}-y_{\kappa, \rho}, y_{\kappa, \rho}-S^{\rho} y_{\kappa, \rho}\right\rangle .
\end{aligned}
$$

Combining (2.9) with (2.10), we arrive at

$$
\alpha\left[1-\left(L_{s}+1\right) \kappa\right]\left\|\bar{x}-S^{\rho} \bar{x}\right\|^{2} \leqslant(\operatorname{diam} C) v_{\rho, s}+\mu_{\rho, s}(\operatorname{diam} C) \psi_{s}((\operatorname{diam} C)), \quad \forall m \geqslant 1 .
$$

Letting $m \rightarrow \infty$, we see that $S^{\rho} \bar{\chi} \rightarrow \bar{x}$. Since $S$ is uniformly $L_{s}$-Lipschitz, we obtain that $\bar{\chi}$ is a fixed point of $S$.

Fix $\kappa^{\prime} \in\left(0, \frac{1}{1+L_{t}}\right)$ and define $y_{\kappa^{\prime}, \rho^{\prime}}=\kappa^{\prime} T^{\rho^{\prime}} \bar{\chi}+\left(1-\kappa^{\prime}\right) \bar{x}$ for arbitrary but fixed $\rho^{\prime} \geqslant 1$. Since both the mappings $S$ and $T$ are Lipschitz continuous, we have

$$
\left\|T^{\rho^{\prime}} x_{n}-x_{n}\right\| \leqslant\left\|T x_{n}-x_{n}\right\|+\left\|T^{2} x_{n}-T x_{n}\right\|+\cdots+\left\|T^{\rho^{\prime}} x_{n}-T^{\rho^{\prime}-1} x_{n}\right\| \leqslant L_{t} K^{\prime}\left\|T x_{n}-x_{n}\right\| .
$$

It follows from (2.6) that

$$
\lim _{n \rightarrow \infty}\left\|T^{\rho^{\prime}} x_{n}-x_{n}\right\|=0
$$

Note that

$$
\begin{aligned}
& \left\langle T^{\rho^{\prime}} y_{\kappa^{\prime}, \rho^{\prime}}-y_{\kappa^{\prime}, \rho^{\prime}}, y_{\kappa^{\prime}, \rho^{\prime}}-\bar{x}\right\rangle=\left\langle x_{n}-y_{\kappa^{\prime}, \rho^{\prime},} y_{\kappa^{\prime}, \rho^{\prime}}-T^{\rho^{\prime}} y_{\kappa^{\prime}, \rho^{\prime}}\right\rangle+\left\langle\bar{x}-x_{n}, y_{k^{\prime}, \rho^{\prime}}-T^{\rho^{\prime}} y_{k^{\prime}, \rho^{\prime}}\right\rangle
\end{aligned}
$$

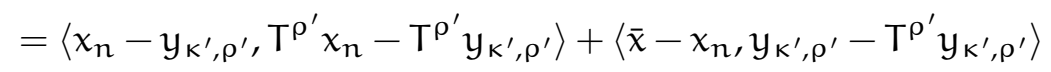

$$
\begin{aligned}
& -\left\langle x_{n}-y_{\kappa^{\prime}, \rho^{\prime}, x_{n}}-y_{\kappa^{\prime}, \rho^{\prime}}\right\rangle+\left\langle x_{n}-y_{\kappa^{\prime}, \rho^{\prime}, x_{n}}-T^{\rho^{\prime}} x_{n}\right\rangle \\
& \leqslant\left\langle x_{n}-\bar{x}, T^{\rho^{\prime}} y_{K^{\prime}, \rho^{\prime}}-y_{\kappa^{\prime}, \rho^{\prime}}\right\rangle \\
& +\left\|x_{n}-y_{k^{\prime}, \rho^{\prime}}\right\|\left(\left\|x_{n}-y_{k^{\prime}, \rho^{\prime}}\right\|+\mu_{\rho^{\prime}, t} \psi_{t}\left(\left\|x_{n}-y_{k^{\prime}, \rho^{\prime}}\right\|\right)+v_{\rho^{\prime}, t}\right) \\
& -\left\|x_{n}-y_{k^{\prime}, \rho^{\prime}}\right\|^{2}+\left\|x_{n}-y_{k^{\prime}, \rho^{\prime}}\right\|\left\|x_{n}-T^{\rho^{\prime}} x_{n}\right\| \\
& \leqslant\left\langle\bar{x}-x_{n}, y_{\kappa^{\prime}, \rho^{\prime}}-T^{\rho^{\prime}} y_{\kappa^{\prime}, \rho^{\prime}}\right\rangle+\left\|x_{n}-y_{\kappa^{\prime}, \rho^{\prime}}\right\|\left\|x_{n}-T^{\rho^{\prime}} x_{n}\right\| \\
& +\mu_{\rho^{\prime}, \mathrm{t}}(\operatorname{diam} \mathrm{C}) \psi_{\mathrm{t}}((\operatorname{diam} \mathrm{C}))+(\operatorname{diam} \mathrm{C}) v_{\rho^{\prime}, \mathrm{t}} \text {. }
\end{aligned}
$$

From (2.11), one has

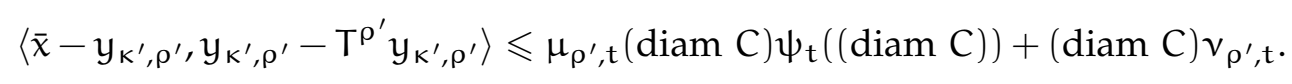

On the other hand, we have

$$
\begin{aligned}
& \kappa\left\|\bar{x}-T^{\rho^{\prime}} \bar{x}\right\|^{2}=\left\langle\bar{x}-y_{\kappa^{\prime}, \rho^{\prime}}, \bar{x}-T^{\rho^{\prime}} \bar{x}\right\rangle
\end{aligned}
$$



$$
\begin{aligned}
& \leqslant\left(L_{t}+1\right) \kappa^{\prime 2}\left\|T^{\rho^{\prime}} \bar{x}-\bar{x}\right\|^{2}+\left\langle\bar{x}-y_{\kappa^{\prime}, \rho^{\prime}}, y_{\kappa^{\prime}, \rho^{\prime}}-T^{\rho^{\prime}} y_{\kappa^{\prime}, \rho^{\prime}}\right\rangle \text {. }
\end{aligned}
$$


Using (2.12) with (2.13), we show that

$$
\alpha\left[1-\left(L_{t}+1\right) \kappa\right]\left\|\bar{x}-T^{\rho^{\prime}} \bar{x}\right\|^{2} \leqslant(\operatorname{diam} C) v_{\rho^{\prime}, t}+\mu_{\rho^{\prime}, t}(\operatorname{diam} C) \psi_{t}((\operatorname{diam} C)), \quad \forall m \geqslant 1 .
$$

Letting $\mathrm{m} \rightarrow \infty$, we see that $\mathrm{T}^{\rho^{\prime}} \overline{\mathrm{x}} \rightarrow \overline{\mathrm{x}}$. Since $\mathrm{T}$ is uniformly $\mathrm{L}_{\mathrm{t}}$-Lipschitz, we obtain that $\bar{x}$ is a fixed point of $\mathrm{T}$.

Finally, we prove that $\left\{x_{n}\right\}$ converges weakly to $\bar{x}$. Assume that there exists some subsequence $\left\{x_{n_{j}}\right\} \subset$ $\left\{x_{n}\right\}$ such that $\left\{x_{n_{j}}\right\}$ converges weakly to $\bar{x}^{\prime} \in C$, where $\bar{x} \neq \bar{x}^{\prime}$. In the same way, we find that $\bar{x}^{\prime} \in$ $\operatorname{Fix}(T) \cap \operatorname{Fix}(S)$. Using the Opial's condition, we find that

$$
d=\lim _{i \rightarrow \infty}\left\|x_{n_{i}}-\bar{x}\right\|<\lim _{i \rightarrow \infty}\left\|x_{n_{i}}-\bar{x}^{\prime}\right\|=\lim _{j \rightarrow \infty}\left\|x_{n_{j}}-\bar{x}^{\prime}\right\|<\lim _{j \rightarrow \infty}\left\|x_{n_{j}}-\bar{x}\right\|=\lim _{i \rightarrow \infty}\left\|x_{n_{i}}-\bar{x}\right\|=d .
$$

This derives a contradiction. It follows that $\bar{x}=\bar{x}^{\prime}$. This shows that $\left\{x_{n}\right\}$ converges weakly to a common fixed point of $S$ and $T$. This completes the proof.

Remark 2.2. Since the class of total asymptotically nonexpansive mappings includes generalized asymptotically nonexpansive mappings, asymptotically nonexpansive mappings in the intermediate sense, asymptotically nonexpansive mappings, and nonexpansive mapping as special cases, Theorem 2.1 is still valid for these nonlinear mappings.

For a single mapping, we have the following result.

Corollary 2.3. Let $\mathrm{C}$ be a nonempty bounded closed and convex subset of a real Hilbert space $\mathrm{H}$. Let $\mathrm{T}: \mathrm{C} \rightarrow \mathrm{C}$ be a uniformly L-Lipschitz and total asymptotically nonexpansive mapping with function $\psi_{\mathrm{t}}$ and nonnegative real sequences $\left\{\mu_{n}\right\}$ and $\left\{v_{n}\right\}$ such that $\sum_{n=0}^{\infty} \mu_{n}<\infty$ and $\sum_{n=0}^{\infty} v_{n}<\infty$. Assume $\operatorname{Fix}(T) \neq \emptyset$ and let $\left\{x_{n}\right\}$ be a sequence generated by the following manner:

$$
\left\{\begin{array}{l}
x_{0} \in C \\
y_{n}=\left(1-\beta_{n}\right) x_{n}+\beta_{n} T^{n} x_{n}, \\
x_{n+1}=\left(1-\alpha_{n}\right) x_{n}+\alpha_{n} T^{n} y_{n}, \quad \forall n \geqslant 0
\end{array}\right.
$$

where $\left\{\alpha_{n}\right\}$ and $\left\{\beta_{n}\right\}$ are two sequences in $(0,1)$ such that $0<a \leqslant \alpha_{n} \leqslant a^{\prime}<1$ and $0<b \leqslant \beta_{n} \leqslant b^{\prime}<1$, where $a, a^{\prime}, b$, and $b^{\prime}$ are four real constants. Then $\left\{x_{n}\right\}$ converges weakly to a fixed point of $\mathrm{T}$.

\section{Strong convergence results}

In this section, we give the strong convergence analysis of the two-step iterative algorithm.

Recall that a mapping $\mathrm{T}: \mathrm{C} \rightarrow \mathrm{C}$ is said to satisfy condition $(A)$ iff there exists a nondecreasing function $f:[0, \infty) \rightarrow[0, \infty)$ with $f(0)=0, f(y)>0$ for all $y \in(0, \infty)$ such that $f(d(x, \operatorname{Fix}(T))) \leqslant\|x-T x\|$, where $d(x, \operatorname{Fix}(T))=\inf \left\{\left\|x-x^{\prime}\right\|: x^{\prime} \in \operatorname{Fix}(T)\right\}$ for all $x \in C$. Maiti and Ghosh [15] and Tan and $X u$ [24] studied the approximation of fixed points of a nonexpansive mapping $\mathrm{T}$ by the Ishikawa iteration under the condition $(A)$ which is weaker than the requirement that $T$ is demicompact.

In this paper, we use the following condition (HC). Let $S, T: C \rightarrow C$ be two mappings. Recall that $\mathrm{T}$ and $\mathrm{S}$ are said to satisfy condition (HC) iff there exists a nondecreasing function $f:[0, \infty) \rightarrow[0, \infty)$ with $f(0)=0, f(y)>0$ for all $y \in(0, \infty)$ such that $f(d(x, \operatorname{Fix}(T))) \leqslant \lambda\|x-T x\|+(1-\lambda)\|x-T x\|$, where $d(x, \operatorname{Fix}(T))=\inf \left\{\left\|x-x^{\prime}\right\|: x^{\prime} \in \operatorname{Fix}(T)\right\}$ for all $x \in C$. Note that condition (HC) reduces to condition $(A)$ in the case that $S=T$.

Theorem 3.1. Let $\mathrm{C}$ be a nonempty bounded closed and convex subset of a real Hilbert space $\mathrm{H}$. Let $\mathrm{T}: \mathrm{C} \rightarrow \mathrm{C}$ be a uniformly $\mathrm{L}_{\mathrm{t}}$-Lipschitz and total asymptotically nonexpansive mapping with function $\psi_{\mathrm{t}}$ and nonnegative real sequences $\left\{\mu_{n, t}\right\}$ and $\left\{v_{n, t}\right\}$ such that $\sum_{n=0}^{\infty} \mu_{n, t}<\infty$ and $\sum_{n=0}^{\infty} v_{n, t}<\infty$. Let $S: C \rightarrow C$ be a uniformly $L_{s^{-}}$ Lipschitz and total asymptotically nonexpansive mapping with function $\psi_{\mathrm{s}}$ and nonnegative real sequences $\left\{\mu_{n, s}\right\}$ 
and $\left\{v_{n, s}\right\}$ such that $\sum_{n=0}^{\infty} \mu_{n, s}<\infty$ and $\sum_{n=0}^{\infty} v_{n, s}<\infty$. Assume $\operatorname{Fix}(T) \cap \operatorname{Fix}(S) \neq \emptyset$ and $S, T$ satisfy condition (HC). Let $\left\{x_{n}\right\}$ be a sequence generated by the following manner:

$$
\left\{\begin{array}{l}
x_{0} \in C \\
y_{n}=\left(1-\beta_{n}\right) x_{n}+\beta_{n} S^{n} x_{n}, \\
x_{n+1}=\left(1-\alpha_{n}\right) x_{n}+\alpha_{n} T^{n} y_{n}, \quad \forall n \geqslant 0
\end{array}\right.
$$

where $\left\{\alpha_{n}\right\}$ and $\left\{\beta_{n}\right\}$ are two sequences in $(0,1)$ such that $0<a \leqslant \alpha_{n} \leqslant a^{\prime}<1$ and $0<b \leqslant \beta_{n} \leqslant b^{\prime}<1$, where $a, a^{\prime}, b$, and $b^{\prime}$ are four real constants. Then $\left\{x_{n}\right\}$ converges strongly to a common fixed point of $\mathrm{T}$ and $\mathrm{S}$.

Proof. From Theorem 2.1, we have that $\lim _{n \rightarrow \infty}\left\|x_{n}-p\right\|$ exists for all $x \in \operatorname{Fix}(S) \cap \operatorname{Fix}(T)$. Without loss of generality, we see that $\lim _{n \rightarrow \infty}\left\|x_{n}-p\right\|=d>0$. From Theorem 2.1, we have that $\lim _{n \rightarrow \infty}\left\|T x_{n}-x_{n}\right\|=$ $\lim _{n \rightarrow \infty}\left\|x_{n}-S x_{n}\right\|=0$ and

$$
\inf _{p \in \operatorname{Fix}(T) \cap \operatorname{Fix}(S)}\left\|x_{n+1}-p\right\| \leqslant \inf _{p \in \operatorname{Fix}(T) \cap \operatorname{Fix}(S)}\left\|x_{n}-p\right\|+\mu_{n, s} \psi_{s}(\operatorname{diam} C)+\mu_{n, t} \psi_{t}(\operatorname{diam} C)+v_{n, t}+v_{n, s} .
$$

That is,

$$
d\left(x_{n+1}, \operatorname{Fix}(T) \cap \operatorname{Fix}(S)\right) \leqslant d\left(x_{n}, \operatorname{Fix}(T) \cap \operatorname{Fix}(S)\right)+\mu_{n, s} \psi_{s}(\operatorname{diam} C)+\mu_{n, t} \psi_{t}(\operatorname{diam} C)+v_{n, t}+v_{n, s} .
$$

Using Lemma 1.1, we have

$$
\lim _{n \rightarrow \infty} d\left(x_{n}, \operatorname{Fix}(T) \cap \operatorname{Fix}(S)\right)
$$

exists. From condition $(H C)$, we have $\lim _{n \rightarrow \infty} f\left(d\left(x_{n}, \operatorname{Fix}(T) \cap \operatorname{Fix}(S)\right)\right)=0$. Since $f$ is nondecreasing with $f(0)=0$, we find that $\lim _{n \rightarrow \infty} d\left(x_{n}, \operatorname{Fix}(T) \cap \operatorname{Fix}(S)\right)=0$.

Next, we take a subsequence $\left\{x_{n_{j}}\right\}$ of $\left\{x_{n}\right\}$ and a sequence $\left\{y_{j}\right\} \subset \operatorname{Fix}(S) \cap \operatorname{Fix}(T)$ such that $\| x_{n_{j}}-$ $y_{j} \|<2^{-j}$. Then, following the method of proof of Tan and $X u$ [24], we get that $\left\{y_{j}\right\}$ is a Cauchy sequence in $\operatorname{Fix}(S) \cap \operatorname{Fix}(T)$ and so it converges. Let $y_{j} \rightarrow y$. Since $\operatorname{Fix}(T) \cap \operatorname{Fix}(S)$ is closed, therefore $y \in \operatorname{Fix}(S) \cap \operatorname{Fix}(T)$ and then $x_{n_{j}} \rightarrow y$. Since $\lim _{n \rightarrow \infty}\left\|x_{n}-p\right\|$ exists for all $x \in \operatorname{Fix}(S) \cap \operatorname{Fix}(T)$, we find that $x_{n} \rightarrow y \in \operatorname{Fix}(S) \cap \operatorname{Fix}(T)$. This completes the proof.

For a single mapping, we have the following result.

Corollary 3.2. Let $\mathrm{C}$ be a nonempty bounded closed and convex subset of a real Hilbert space $\mathrm{H}$. Let $\mathrm{T}: \mathrm{C} \rightarrow \mathrm{C}$ be a uniformly $\mathrm{L}_{\mathrm{t}}$-Lipschitz and total asymptotically nonexpansive mapping with function $\psi_{\mathrm{t}}$ and nonnegative real sequences $\left\{\mu_{n, t}\right\}$ and $\left\{v_{n, t}\right\}$ such that $\sum_{n=0}^{\infty} \mu_{n, t}<\infty$ and $\sum_{n=0}^{\infty} v_{n, t}<\infty$. Assume $\operatorname{Fix}(T) \neq \emptyset$ and $T$ satisfies condition $(A)$. Let $\left\{x_{n}\right\}$ be a sequence generated by the following manner:

$$
\left\{\begin{array}{l}
x_{0} \in C \\
y_{n}=\left(1-\beta_{n}\right) x_{n}+\beta_{n} T^{n} x_{n}, \\
x_{n+1}=\left(1-\alpha_{n}\right) x_{n}+\alpha_{n} T^{n} y_{n}, \quad \forall n \geqslant 0
\end{array}\right.
$$

where $\left\{\alpha_{n}\right\}$ and $\left\{\beta_{n}\right\}$ are two sequences in $(0,1)$ such that $0<a \leqslant \alpha_{n} \leqslant a^{\prime}<1$ and $0<b \leqslant \beta_{n} \leqslant b^{\prime}<1$, where $\mathrm{a}, \mathrm{a}^{\prime}, \mathrm{b}$, and $\mathrm{b}^{\prime}$ are four real constants. Then $\left\{\mathrm{x}_{\mathrm{n}}\right\}$ converges strongly to a fixed point of $\mathrm{T}$.

Finally, we give two numerical examples to illustrate the efficient of the algorithm.

Suppose that $H:=R$ and $C:=[0,1] \subset R$. Define a mapping $T$ by $T x=k x, \forall x \in\left[0, \frac{1}{2}\right]$ and $T x=0$, $\forall x \in\left(\frac{1}{2}, 1\right]$, where $k$ is a constant in $(0,1)$. Then we observe that $F(T)=0$, and hence the set of the fixed points is nonempty.

Now, we show that $T$ is total asymptotically pseudocontractive mapping. Suppose that $C_{1}=\left[0, \frac{1}{2}\right]$ and $\mathrm{C}_{2}=\left(\frac{1}{2}, 1\right]$ 
Case 1. If $x, y \in C_{1}$, then $\left|T^{n} x-T^{n} y\right|=\left|k^{n} x-k^{n} y\right| \leqslant|x-y|$.

Case 2. If $x, y \in C_{2}$, then $\left|T^{n} x-T^{n} y\right| \leqslant|x-y|$.

Case 3. If $x \in C_{1}, y \in C_{2}$, then $\left|T^{n} x-T^{n} y\right| \leqslant\left|k^{n} x-0\right| \leqslant k^{n}|x-y|+k^{n}|y| \leqslant|x-y|+k^{n} M$, where $M$ is some real constant. If we choose $x_{0} \in C$ arbitrarily, then for four different initial values, we see all the results are convergent in Figure 1 . Let $D=[-1,1], D_{1}=[-1,0]$, and $D_{2}=[0,1]$. If we choose $x_{0} \in \mathrm{D}$ arbitrarily, then for four different initial values, we see all the results are convergent in Figure 2.

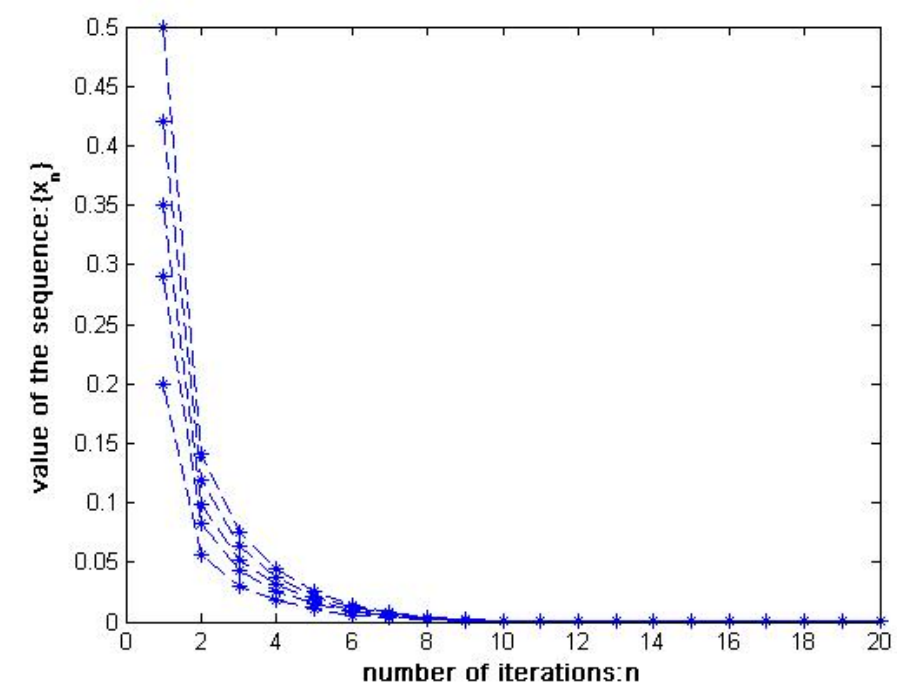

Figure 1

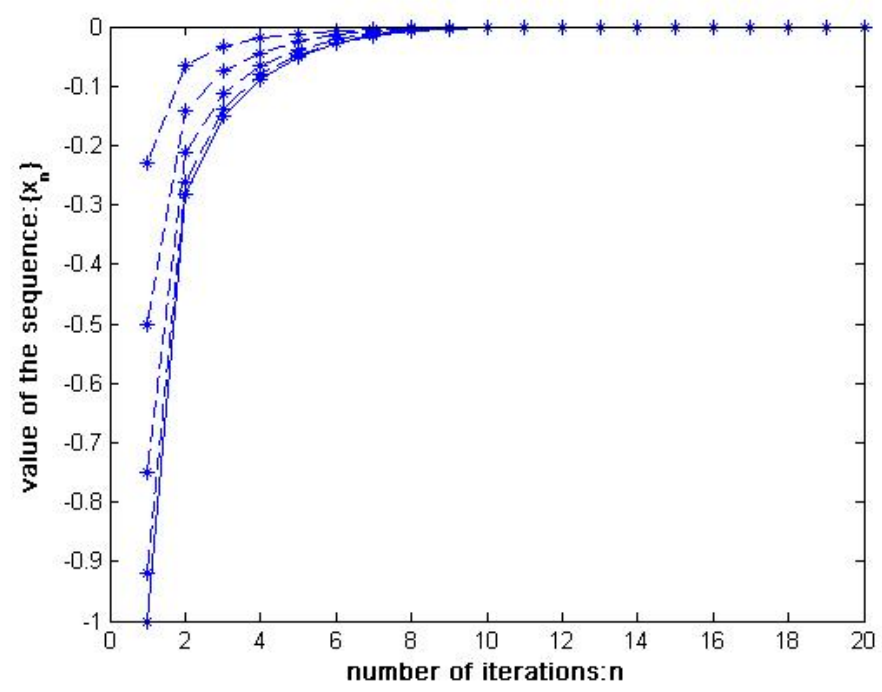

Figure 2

\section{Acknowledgment}

The authors would like to thank the editor and the referee for useful suggestions which improve the contents of this article. 


\section{References}

[1] R. P. Agarwal, X.-L. Qin, S. M. Kang, An implicit iterative algorithm with errors for two families of generalized asymptotically nonexpansive mappings, Fixed Point Theory Appl., 2011 (2011), 17 pages. 1

[2] Y. I. Alber, C. E. Chidume, H. Zegeye, Approximating fixed points of total asymptotically nonexpansive mappings, Fixed Point Theory Appl., 2006 (2006), 20 pages. 1

[3] I. K. Argyros, S. George, S. M. Erappa, Expanding the applicability of the generalized Newton method for generalized equations, Commun. Optim. Theory, 2017 (2017), 12 pages. 1

[4] B. A. Bin Dehaish, A. Latif, H. O. Bakodah, X.-L. Qin, A regularization projection algorithm for various problems with nonlinear mappings in Hilbert spaces, J. Inequal. Appl., 2015 (2015), 14 pages. 1

[5] R. Bruck, T. Kuczumow, S. Reich, Convergence of iterates of asymptotically nonexpansive mappings in Banach spaces with the uniform Opial property, Colloq. Math., 65 (1993), 169-179. 1

[6] C. Byrne, A unified treatment of some iterative algorithms in signal processing and image reconstruction, Inverse Problems, 20 (2014), 103-120. 1

[7] S. Y. Cho, B. A. Bin Dehaish, X.-L. Qin, Weak convergence of a splitting algorithm in Hilbert spaces, J. Appl. Anal. Comput., 7 (2017), 427-438. 1

[8] S. Y. Cho, W.-L. Li, S. M. Kang, Convergence analysis of an iterative algorithm for monotone operators, J. Inequal. Appl., 2013 (2013), 14 pages. 1

[9] N.-N. Fang, Y.-P. Gong, Viscosity iterative methods for split variational inclusion problems and fixed point problems of a nonexpansive mapping, Commun. Optim. Theory, 2016 (2016), 15 pages. 1

[10] A. Genel, J. Lindenstrauss, An example concerning fixed points, Israel J. Math., 22 (1975), 81-86. 1

[11] K. Goebel, W. A. Kirk, A fixed point theorem for asymptotically nonexpansive mappings, Proc. Amer. Math. Soc., 35 (1972), 171-174. 1

[12] Z.-Y. Huang, Mann and Ishikawa iterations with errors for asymptotically nonexpansive mappings, Comput. Math. Appl., 37 (1999), 1-7. 1

[13] S. H. Khan, H. Fukhar-ud-din, Weak and strong convergence of a scheme with errors for two nonexpansive mappings, Nonlinear Anal., 61 (2005), 1295-1301. 1

[14] J. K. Kim, S. Y. Cho, X.-L. Qin, Some results on generalized equilibrium problems involving strictly pseudocontractive mappings, Acta Math. Sci. Ser. B Engl. Ed., 31 (2011), 2041-2057. 1

[15] M. Maiti, M. K. Ghosh, Approximating fixed points by Ishikawa iterates, Bull. Austral. Math. Soc., 40 (1989), $113-117$. 3

[16] Z. Opial, Weak convergence of the sequence of successive approximations for nonexpansive mappings, Bull. Amer. Math. Soc., 73 (1967), 591-597. 1

[17] H. Piri, R. Yavarimehr, Solving systems of monotone variational inequalities on fixed point sets of strictly pseudocontractive mappings, J. Nonlinear Funct. Anal., 2016 (2016), 18 pages. 1

[18] X.-L. Qin, S. Y. Cho, Convergence analysis of a monotone projection algorithm in reflexive Banach spaces, Acta Math. Sci. Ser. B Engl. Ed., 37 (2017), 488-502. 1

[19] X.-L. Qin, S. Y. Cho, L. Wang, A regularization method for treating zero points of the sum of two monotone operators, Fixed Point Theory Appl., 2014 (2014), 10 pages. 1

[20] X.-L. Qin, J.-C. Yao, Weak convergence of a Mann-like algorithm for nonexpansive and accretive operators, J. Inequal. Appl., 2016 (2016), 9 pages. 1

[21] S. Reich, Weak convergence theorems for nonexpansive mappings in Banach spaces, J. Math. Anal. Appl., 67 (1979), 274-276. 1

[22] J. Schu, Iterative construction of fixed points of asymptotically nonexpansive mappings, J. Math. Anal. Appl., 158 (1991), 407-413. 1

[23] J. Schu, Weak and strong convergence to fixed points of asymptotically nonexpansive mappings, Bull. Austral. Math. Soc., 43 (1991), 153-159. 1, 1.2

[24] K.-K. Tan, H.-K. Xu, Approximating fixed points of nonexpansive mappings by the Ishikawa iteration process, J. Math. Anal. Appl., 178 (1993), 301-308. 1.1, 3, 3

[25] H. Zegeye, W. W. Kassu, M. G. Sangago, Common fixed points of a finite family of multi-valued rho-nonexpansive mappings in modular function spaces, Commun. Optim. Theory, 2016 (2016), 14 pages. 1 